\title{
The Constraining of Parameters in Restricted Factor Analysis
}

\author{
John Hattie \\ University of Western Australia \\ Colin Fraser \\ Contre for Behavioural Studies in Education, Ammidale, Australia
}

In restricted factor analysis each of the elements in the matrices (of factor loadings, factor correlations, and unique variances and covariances) can be fixed, free to be estimated, or constrained. In this paper it is argued that the common practice of constraining some parameters at 0 often is not psychologically meaningful. Alternative procedures for constraining parameters are presented and illustrated using data based on the Luria model. Index terms: Constrained parameters, Factor analysis, Factor correlations, Factor loadings, Factor uniqueness.

Many applications of factor analysis use procedures such as the analysis of covariance strucures (e.g., Jöreskog, 1969, 1978; McDonald, 1978 , 1980), especially using the LISREL computer program (Jöreskog \& Sörbom, 1978). These methods have been heralded as "perhaps the most important and influential statistical revolution to have occurred in the social sciences"' (Cliff, 1983, p. 115). Yet Cliff also argued that these methods may instead "become a disaster" because "they seem to encourage [the researcher] to suspend his normal critical faculties" (p. 116). He then detailed four reasons for this presentiment: that data do not confirm a model, they only fail to disconfirm it; that an assumed cause-and-effect relationship may not actually exist; that just because researchers name factors, this does not mean that they understand

APPLIED PSYCHOLOGLCAL MEASUREMEN7

Vol. 12, No. 2, June 1988, pp. 155-162

(C) Copyright 1988 Applied Psychological Measurement Inc. 0146-6216/88/020155-08\$1.65 them, or even that factors have been named correctly; and that hindsight can be unreliable. (Because of these reasons and others, the generic name "restricted" is preferred over "confirmatory" facfor analysis.)

Even before the analyses commence, problems can occur relating to the choice of items. In an attempt to create a set of items with a single underlying dimension or common factor, some researchers might seleci irems that differ from each other only by the use of symonyms or by paraphrasing. Clearly, this effort would be fruitless. Further, if should not be surprising that a particular factor does not emerge from an analysis of a set of items if there are insufficient variables in the set to define that factor.

Once the analysis begins, the researcher can make poor judgments, for example regarding the choice of computer programs (MacCallum, 1983). Usually these errors in judgment are a consequence of depending upon "defaul" options. These options are not suitable for every dataset to be analyzed. For example, a common option of questionable merit is the choice of eigenvalues greater than 1 to determine the number of factors. The researcher should examine carefully the defaults in a computer program and, if necessary, make substitutions based on a knowledge of the theory underlying the data.

A default commonly used in restricted factor analysis is the use of Os as constrained parameters. Some background is necessary to illustrate this problem. The usual factor-analytic model can be 
represented by the matrix equation

$\mathbb{C}=\mathbb{F S F}^{\prime}+\mathbb{U}^{2}$,

where $\mathbb{C}$ is the covariance of correlation matrix, $F$ is the matrix of factor loadings, $\mathbb{S}$ is the matrix of correlations between factors, and $U$ contains the unique variances and covariances. In many unrestricted factor analyses, only the factor loadings and unique variances are reported because of two common defaults: (1) an orthogonal (e.g., Vari$\max$ ) solution, where $\mathbb{S}$ is the identity matrix, and (2) the assumption that $U$ is a diagonal matrix, implying that the unique covariances are all 0 .

In restricted factor analysis, the matrices of $F$, $\mathbb{S}$, and $\mathbb{U}$ can be patterned so that each of their elements can be fixed, free to be estimated, or constrained. The most common use is to pattern $F$ so that the variables are allowed to load only on a particular factor, to allow the off-diagonal elements of $\mathbb{S}$ to be estimated, and to estimate $\mathbb{U}$ as a diagonal matrix. As MicDonald (1980) has shown, patterning embodies most null hypotheses of interest in multivariate analysis, "namely that certain covariances, variances, correlations or regression weights are zero or equal" to one another (p. 162).

Typically, researchers have set up hypotheses relating to the patterning in the data by setting some elements to 0 and leaving some elements free to be estimated. The fixed elements, however, do not have to be 0 , and there are occasions when it is psychologically meaningfui to allow the fixed values to be other than 0 . The major purpose of this paper is to give instances of the inappropriate use of os and to present methods for estimating nonzero constrained elements.

\section{Constraining the Factor Loadings}

In restricted factor analysis, a factor loading is fixed at 0 if it is believed that the corresponding factor does not contribute to that variable. For example, a battery of marker tests has been developed to identify three factors: simultaneous processing, successive processing, and attention. These factors are central to the model of information processing proposed by Luria (1978).

The tests used to identify simultaneous process- ing usually involve the ability to reason with images of spatial arrays without altering the relationships between components. An example of such a test is the Matrix rotation test (Fitzgerald, 1971). Fony sets are drawn of a $3 \times 3$ dot matrix and the test requires the retention and recall of these visual patterns, or their mental manipulation. The first 20 items are presented for 5 seconds, and then the examinee is allowed 10 seconds to reproduce the shape on a supplied empty dot matrix. The second 20 items are presented for 15 seconds and then the examinee is asked to mentally rotate the shape 180 degrees before reproducing it.

The tests used to identify successive processing involve stimuli that evoke a particular chain of successive links. An example is letter span (Ekstrom, French, \& Harman, 1976). This test consists of a series of letters presented visually, and the examinee is asked to write them down in original order at the end of presentation. The size of the series (from 4 to 11 letters) varies randomly over 20 presentations. The scoring emphasizes the maintenance of correct serial order within the series. The final factor is attention, and the tests used involve selecting essential elements in a processing task. A typical task presents a mixed series of letters or numbers, and at the end of presentation the examinee is asked to recall either the letters or the numbers. The scoring is also based on the correct serial order of recall.

The present authors' research over the past 10 years has investigated a variety of measures; a major aim has been to locate measures of simultaneous and successive processing that are uncorrelated. Given Luria's theories, it is not hypothesized that attention measures are uncorrelated with the simultaneous and successive factors.

The remainder of this paper uses restricted factor analysis based on six datasets. Each set is based on different marker tests for the three factors. For reasons that will become clearer, the computer program COSAN (Fraser, 1982) was used, rather than the more widely available LISREL.'

A reviewer has pointed out that the BMDP program EQS also handles the inequality constraints discussed in this paper. 
Table 1

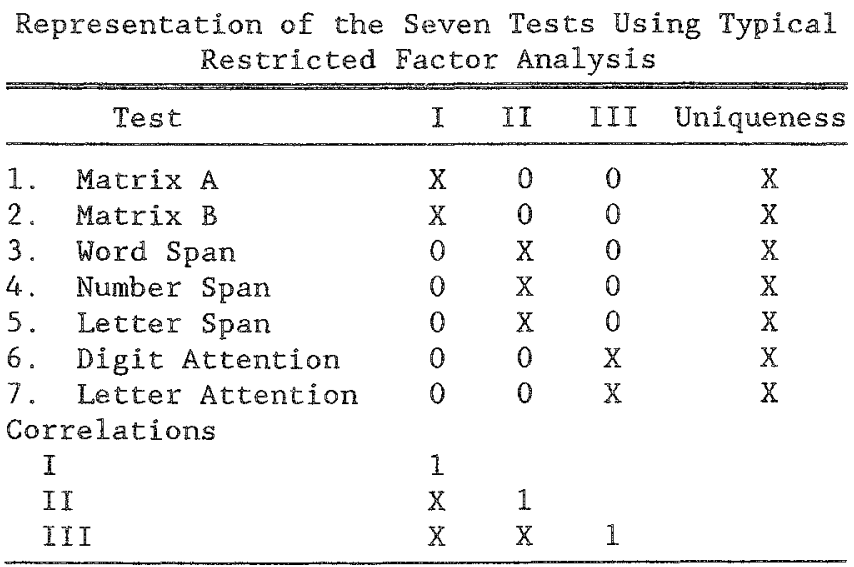

Without considering the detailed context of the variables, the patterning illustrated in Table 1 might appear to be reasonable. The three factors were labeled as simultaneous processing, successive processing, and attention span. The $X$ s indicate elements that are free to be estimated, whereas the $O s$ and $I s$ are to be fixed at those values. Because $\mathbb{U}$ is patterned as a diagonal matrix of unique variances, only those elements are listed in Table 1. Analysis of this patterning produced the summary fit statistics for each dataset; these are presented in the first row in Table 2.

Constraining the fixed factor loadings to exactly 0 , however, is not consonant with knowledge of how these dimensions should theoretically relate to each other (see Das, Kirby, \& Jarman, 1979; Klich, 1983; Walton, 1983). From Luria's theory, it is expected that the successive tests will have nonzero estimates on the attention factor. Consequently, rather than allowing the values for these successive tests free to be estimated, it is desired that the loadings be small but not constrained exactly to 0 . It is more reasonable to constrain the values to be bounded, for example between -.25 and .25 . Another method of constraint is ordering the parameters by magnitude (e.g., for the Luria data it might be specified that on factor 1 , variables 1 and 2 should have loadings greater than the other five variables).

Both types of constraints can be handled by reparamcterization within $\operatorname{COSAN}$. When a parameter is unconstrained it may take on any value in the interval $(-\infty,+\infty)$. To impose bounds to constrain a parameter within the interval $(a, b)$, estimate the quantity

$\lambda=\frac{(b-a)}{2} \sin \theta+\frac{(b+a)}{2}$

in place of the free parameter $\theta$. McDonald (1980) suggested another method to impose bounds and also proposed a method to impose an ordering on the parameters so that, for example, $\lambda_{1} \leqslant \lambda_{2} \leqslant \ldots$ $\lambda_{k}$. It is possible to reparameterize by estimating

$\lambda_{2}=\lambda_{1}+\theta_{2}^{2}$

.

$\lambda_{k}=\lambda_{k-1}+\theta_{k}^{2}$

Other kinds of reparameterizations are possible. When using COSAN, the researcher may use any type of constraint, provided the corresponding reparameterizations can be provided as shown above. These methods to impose bounds to a constrained parameter also could be used to constrain the common variance to be no greater than permitted by the reliability (see Rindskopf, 1983).

The Luria data were reanalyzed by using the first type of constraint mentioned above: Some of the factor loadings were constrained to be in the interval $(-.25,25)$ and the remaining constrained values were set to 0 . These values must be chosen 


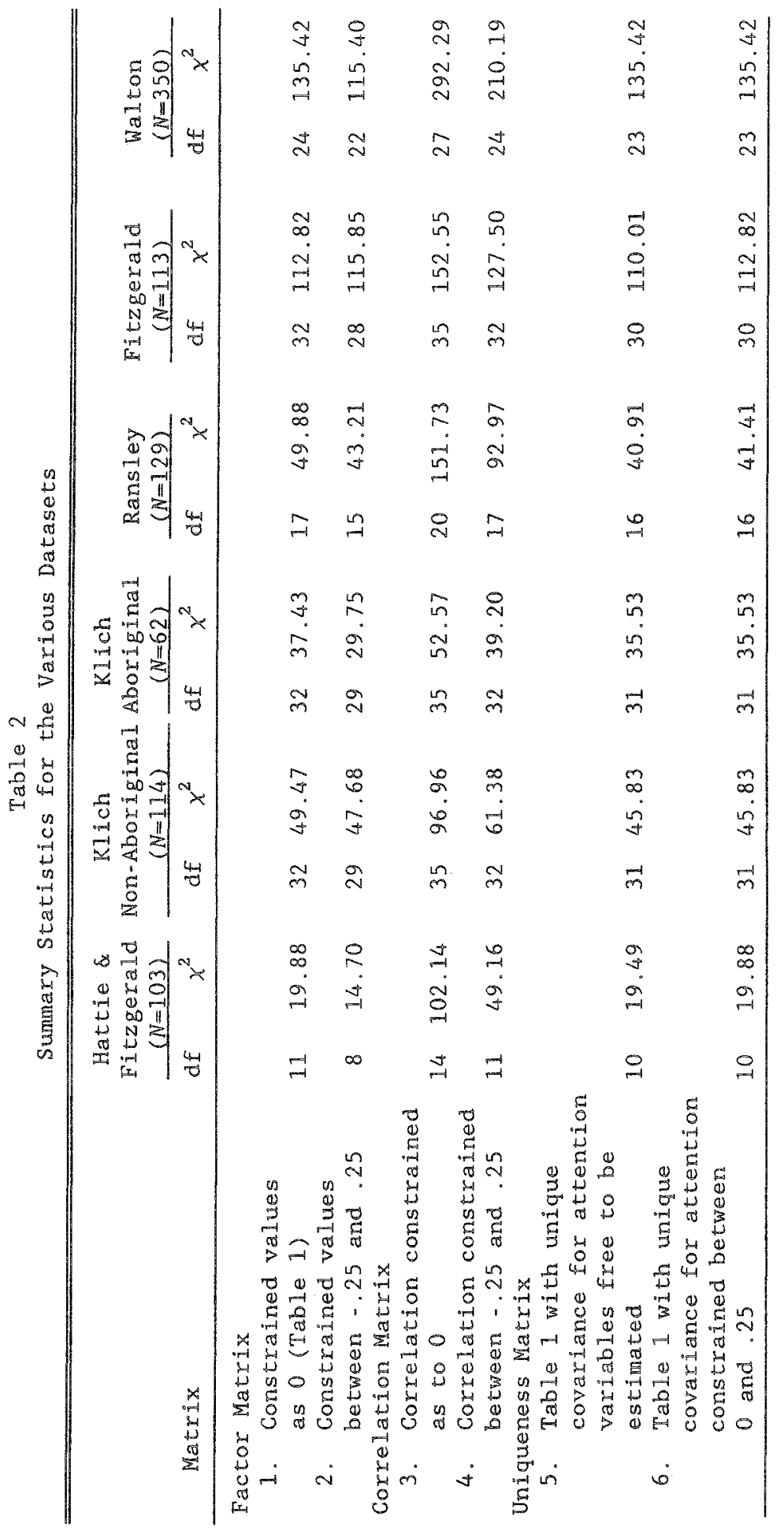


on theoretical grounds. The aim in the present case was to develop measures of simultaneous and successive processing that were independent of each other. Given that a "test" does not completely dictate the person's style of processing, it was realized that some successive tests may require some simultaneous processing (and vice versa).

The values of \pm .25 seemed a reasonable compromise between a zero and a meaningful salientloading. Unfortunately, it is not yet possible to test inequalities for statistical necessity (but see Shapiro, 1985, for some suggestions). Further defense of these bounds is outside the scope of this paper and, although reasonable, will serve for illustration purposes only. Obviously, different values may be more appropriate in other situations, and the choice of values must depend on theoretical considerations.

In Table 2, the chi-square value for the model with no range constraints is presented first to enable comparisons with models with range constraints. There was a significant improvement in only two of the six cases - for the Walton (1983) and Ransley (1981) data - when large constraints were added to the factor structure. The results of these analyses are presented in the second row in Table 2.

\section{Comstraำing 娟erwen Tactors}

It has not been possible to find a published example of a restricted factor analysis wherein the factors are uncorrelated, that is, where $\mathbb{S}$ is fixed as an identity matrix. There are many psychological theories, however, that assume that the factors are uncorrelated. A simple example will illustrate why it is not usual to find examples of uncorrelated factors. Suppose $\$$ is an identity matrix and has the simple structure pattern

$$
\left[\begin{array}{ll}
x & 0 \\
x & 0 \\
x & 0 \\
0 & x \\
0 & x \\
0 & x
\end{array}\right]
$$

Then $\mathbb{F S}^{\prime} \mathbf{F}^{\prime}=\mathbb{F}^{\prime}$ will have the pattern

$$
\left[\begin{array}{llllll}
x & x & x & 0 & 0 & 0 \\
x & x & x & 0 & 0 & 0 \\
x & x & x & 0 & 0 & 0 \\
0 & 0 & 0 & x & x & x \\
0 & 0 & 0 & x & x & x \\
0 & 0 & 0 & x & x & x
\end{array}\right]
$$

If $U$ is a diagonal matrix, any difference between the estimated $\mathbf{C}\left(=\mathbb{F S} \mathbb{F}^{\prime}+\mathbb{U}^{2}\right)$ and the observed correlation matrix will have the observed correlations as residuals wherever there are 0 s. If correlated factors are allowed, the fit will be much better.

It is possible, however, to combine uncorrelated factors and the methods outlined above, imposing bounds around the fixed values in the factor loading matrix to produce reasonable fit. It is also possible to constrain the correlations between factors such that they are between -.25 and .25 . The summary statistics for both an orthogonal model and a constrained model $(-.25, .25)$ are presented in Table 2.

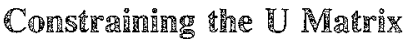

Typically, the U matrix has parameters in the diagonal that correspond to the unique variances, and fixed os elsewhere that correspond to the unique covariances. The unique variances, or uniquenesses, represent the amount of variance in a given variable that is not accounted for by the common factors.

A problem encountered with estimating uniquenesses is that zero or negative estimates can arise. When this happens, the corresponding variables are termed Heywood cases and they indicate that an improper solution has been found and/or that the variable has no measurement error. Further, when estimating the off-diagonal unique covariances, the resulting $U$ matrix should be positive definite. It is possible to formulate the model for COSAN so that the resulting $\mathrm{U}$ matrix is non-negative definite, thus ensuring non-negative uniquenesses. Instead of estimating the $U$ matrix directly, a lower triangular matrix $W$ can be estimated and $U=W W^{\prime}$, which by definition is non-negative definite, can be com- 
Volume 12 Number 2 June 1988

160
APPLED PSYCHOLOGICAL MEASUREMENT

puted. If the unique covariances need not be estimated, $W$ is a diagonal matrix and $U=\mathbb{W}^{2}$.

Of particular interest is the estimation of the offdiagonal unique covariances. Typically these are fixed at 0 . However, these elements could be estimated in some models. Examples include multimode factor analysis (Law, Snyder, Hattie, \& McDonald, 1984), longitudinal factor analysis (see McDonald, 1980; Sörbom, 1975), and cases where an item in a test contributes to different scales (such as in the MMPI or POI; see Hattie, 1981) or where two scores are derived from one item (as in many creativity rests; see Hattie \& Rogers, 1986).

In the Luria data, the attention scores (Digit Attention and Letter Attention) are obtained from one administration of 20 items. For each item a mixture of letters and numbers is presented (e.g., $L 8 \mathrm{~T} \mathrm{~V}$ 365 ) and the examinee is asked to recall either the letters or the numbers. Thus, the unique covariance between the two scores may not be 0 . The datasets were reanalyzed allowing the unique covariance between the attention tests to be estimated at any value (and, for the completeness of the examples, constrained between 0 and .25). Only a trivial improvement in the fit of the four models occurred, and the average unique covariance was small (.17). The summary statistics for these analyses are presented in Table 2 .

\section{Conchusions}

Restricted factor analysis is becoming an influential and commonly used procedure. The procedure is mathematically quite sophisticated and may confuse the naive user, who may assume that certain defaults available in commonly used computer programs are appropriate and correct for every purpose.

A major issue is deciding which parameters should be free, fixed, or constrained and, if constrained, what type of constraint should be used. The type of constraint includes imposing bounds, specifying values, and ordering parameters. These decisions depend critically on theoretical a priori justifications. There is little scientific merit in the present practice of deciding, after an analysis, which pa- rameters should be constrained (and how they should be constrained) so as to improve fit. Brynner (1981), for example, tried to fit a series of models after successively inspecting the fit and residuals. He claimed that indications of the adequacy of the model are obtained by removing the zero constraints "one by one and observing the effect on the maximum likelihood goodness-of-fit values" (p. 524). These attempts have little justification.

Such pursuits violate good social science methodology (Cliff, 1983). Many alternative models fit the data, and many of these models will fit the data equally well. As argued elsewhere (see Bentler \& Bonett, 1980; Hattie, 1981), it is probable that testing competing theoretical models may lead to more advances than merely aftempting to "not reject" a single model. Yet competing theoretical models are not to be confused with models that have been successively improved after inspection of residual matrices, chi-squares, factor loadings, or standard errors. This is not to eschew exploratory analysis. Much justification exisis for exploring, say, half the data so long as they are crossvalidated and preferably double cross-validated (Hattie, 1981; Mosteller \& Tukey, 1968, 1977).

If the researcher does have competing nested models, then it is possible to use the difference in chi-squares test (Bentler \& Bonett, 1980). These differences do not take into account that the probabilities from the chi-squares come from the same sample, and it is not certain that the degrees of freedom are correct (see McDonald \& Krane, 1979). Rindskopf (1983) suggested that when constraints such as suggested above were introduced, an additional degree of freedom could be counted for each active constraint.

Schmitt (1978) suggested that a $\chi^{2 / d f}$ ratio larger than 10 indicates an inadequate fit, while a ratio less than 1 indicates a model fits "too well." A model that fits too well, claimed Schmitt, could be unstable in future samples. In the present example, all but one model fits adequately. Yet this ratio provides no information about the relative fit, nor does there seem to be any justification for the index.

Alternative and justifiable methods for comparing various models have been advanced by Bentler 
and Bonett (1980) and Buse (1982). Their index is calculated as

$$
p_{21}=\frac{\frac{\chi_{2}^{2}}{d f_{2}}-\frac{\chi_{1}^{2}}{d f_{1}}}{\frac{\chi_{0}^{2}}{d f_{0}}-1},
$$

where $\chi_{1}^{2}$ and $\chi_{2}^{2}$ are competing models and $\chi_{0}^{2}$ is based on the general null model of 0 common factors. In the present example, all solutions are a substantial improvement over the null model. There were trivial differences between the models, however, provided the values in the off-diagonals of the correlation matrix were free to be estimated.

There are many problems in deciding how to constrain parameters in restricted factor analysis. Allowing the constrained values to be 0 (as is commonly done, by default) involves as much of a theoretical decision as selecting nonzero value(s). The methods outlined in this paper illustrate the means to calculate constrained values, and it must be emphasized that the placement and the choice of values depend on theoretical concerns. The present authors agree with Cliff's (1983) statement that restricted factor analysis may be a disaster if the methods encourage suspension of normal critical faculties.

\section{Referereres}

Bentler, P. M., \& Bonett, D. G. (1980). Significance tests and goodness of fit in the analysis of covariance structures. Psychological Bulletin, 88, 588-606.

Brynner, J. (1981). Use of LISREL in the solution to a higher-order factor problem in a study of adolescent self-images. Quality and Quantity, 15, 523-540.

Buse, A. (1982). The likelihood ratio, Wald, and Lagrange Multiplier tests: An expository note. The American Statistician, 36, 153-157.

Cliff, N. (1983). Some cautions concerning the application of causal modeling methods. Multivariate Behavioral Research, 18, 115-126.

Das, J. P., Kirby, J., \& Jarman, R. F. (1979). Simultaneous and successive cognitive processes. New York: Academic Press.

Ekstrom, R. B., French, J. W., \& Harman, H. H. (1976). Manual for the Kit of Factor-Referenced Cognitive Tests. Princeton NJ: Educational Testing Service.

Fiøzgerald, D. (1971). A matrix test for measuring simultaneous processing. Unpublished report, Division of Educational Research Services, University of Alberta.

Fraser, C. (1982). COSAN: A FORTRAN program for covariance structures analysis of the Nth order [Unpublished computer program]. Armidale, NSW, Australia: University of New England.

Hattie, J. A. (1981). A four-stage factor-analytic approach to studying behavioral domains. Applied Psychological Measurement, 5, 77-88.

Hattie, J. A., \& Rogers, H. J. (1986). Factor models for assessing the relation between creativity and intelligence. Journal of Educational Psychology, 78, $482-485$.

Jöreskog, K. G. (1969). A general approach to confirmatory factor analysis. Psychometrika, 34, 183-202.

Jöreskog, K. G. (1978). Structural analysis of covariance and correlation matrices. Psychometrika, 43, 443477.

Jöreskog, K. G., \& Sörbom, D. (1978). LISREL-Analysis of linear structural relationships by the method of maximum likelihood. Chicago: National Educational Resources Inc

Klich, L. Z. (1983). A cross-cultural examination of cognitive functions based on Luria's neuropsychological theory of cerebral organization. Unpublished doctoral dissertation, University of New England, Australia.

Law, H., Snyder, W., Hattie, J. A., \& McDonald, R. P. (1984). Research methods for multi-mode data analysis in the behavioral sciences. New York: Praeger.

Luria, A. R. (1978). The working brain. Middlesex: Penguin.

MacCallum, R. (1983). A comparison of factor analysis programs in SPSS, BMD, and SAS. Psychometrika, 48 , 223-23!

McDonald, R. P. (1978). A simple comprehensive model for the analysis of covariance structures. British Journal of Mathematical and Statistical Psychology, 31 , 59-72.

McDonald, R. P. (1980). A simple comprehensive model for the analysis of covariance structures: Some remarks on applications. British Journal of Mathematical and Statistical Psychology, 33, 161-183.

McDonald, R. P., \& Krane, W. P. (1979). A monte carlo study of local identifiability and degrees of freedom in the asymptotic likelihood ratio test. British Journal of Mathematical and Statistical Psychology, $32,121-132$.

Mosteller, F., \& Tukey, J. W. (1968). Data analysis including statistics. In G. Lindzey \& E. Aronson (Eds.), The handbook of social psychology ( 2 nd ed.). Reading MA: Addison-Wesley.

Mosteller, F., \& Tukey, J. W. (1977). Data analysis and regression. Reading MA: Addison-Wesley. 
Ransley, W. K. (1981). The development of a psychometric model of information processing in young children based on Luria's theory of brain functioning. Unpublished doctoral dissertation, University of New England, Australia.

Rindskopf, D. (1983). Parameterizing inequality constraints on unique variances in linear structure models. Psychometrika, 48, 73-83.

Schmitt, N. (1978). Path analysis of multitrait-multimerhod matrices. Applied Psychological Measurement, 2, 157-173.

Shapiro, A. (1985). Asymptotic distribution of test staristics in the analysis of moment structures under inequality constraints. Biometrika, 72, 133-144.

Sörbom, D. (1975). Detection of correlated errors in longitudinal data. British Joumal of Mathematical and Statistical Psychology, 28, 138-151.
Walton, J. E. (1983). Sequential and simultaneous information processing abilities and their interaction with instructional treatments in senior high school mathematics. Unpublished doctoral dissertation, University of New England, Australia.

\section{Acknowedgments}

The authors thank Gil Sax for his helpful comments.

\section{Aathor's Address}

Send requests for reprints or further information to John Hattie, Department of Education, University of Western Australia, Nedlands, Western Australia 6009, Australia. 